\title{
THE IAU NUTATION THEORY AND PERSPECTIVES OF
}

\section{ITS CHANGE.}

D. MCCARTHY

U.S. Naval Observatory, Washington DC 20392, USA

AND

V. DEHANT

Royal Observatory of Belgium

3, avenue Circulaire, B-1180 Brussels, Belgium

\section{Present situation}

The IAU General Assembly has adopted in 1980 a nutation series, on the one hand, based on rigid Earth's contributions theorically computed from celestial mechanics, and on the other hand, based on non-rigid Earth's contributions theoretically computed from Earth deformation equations using geophysical parameters.

1. From the previous papers (see Session 1) of this Joint Discussion, we know that there are differences of this adopted theory with respect to the observations of precession and nutations. These differences can reach several mas, which is well above the present accuracy of the observations.

2. From previous papers (see Session 2) and from the posters, we also know that there exist new rigid-Earth nutations (Kinoshita-Souchay, Roosbeek, Hartmann) of which the accuracy has increased by one order of magnitude.

3. From Session 3 papers, we know that there are some additional geophysical effects that are not yet taken into account in Wahr's nutation theory adopted in 1980 by the IAU which have a contribution at a level above the present precision of the observations. These additional geophysical aspects can be accounted for either from a semi-analytical theory (like Mathews, Herring, Shapiro and Buffett are doing), or from 
an integration of deformation equations through the whole Earth (Dehant, Wahr).

4. In Session 1 of our JD we also learned that there exist an empirical model based, on the one hand, on VLBI (and LLR) nutation observations, and on the other hand on a geophysically based resonance formula.

\section{Decision for the future among the following choices:}

1. Continue to use the IAU 1980 nutation series? This is possible if the precision required is not better than 10 mas, but for most practical uses, a better precision is needed.

2. Use observed nutation series from the IERS (combination of LLR and VLBI data)? This would be possible but :

- there are observational errors

- there are correlations between long-period (e.g. 18.6 yr ) nutations in longitude and precession,

- there are correlations between long-period (e.g. $18.6 \mathrm{yr}$ ) nutations in obliquity and obliquity rate.

3. Use a complete theoretical nutation series based on celestial mechanics and geophysics? This series is not yet available.

4. Use semi-observed, semi-theoretical nutation series? i.e.:

- use Kinoshita-Souchay rigid Earth nutation series,

- use the resonance formula with recent theoretical results,

- EXCEPT for some "geophysical problem"-nutations like the annual nutations or the $18.6 \mathrm{yr}$ nutations, for which observed amplitudes are used.

We think that there is a danger for mixing observation and theory. Moreover, additional geophysical corrections from semi-analytical theory (as Mathews for instance) and numerical integration (as Dehant for instance) are not yet in a total agreement.

5. Use an empirical model based on Kinoshita-Souchay's rigid-Earth nutations and on a resonace formula where the parameters are determined from IERS nutation observations? This would be the best choice because of its simplicity in comparison with the other possibilities. This would provide us with an "a-priori" model for precession and nutation as it is needed for practical use. But be careful not to interpret this formula in terms of geophysics. 